\title{
SCRIVERE TRA DUE LINGUE: Lingua ITALiana E Traduzione in Christiana de Caldas Brito e VERA LúCIA DE Oliveira
}

\section{Pierangela Di Lucchio}

$\Lambda$ BSTRACT Questo lavoro si articola in un'analisi comparativa tra alcuni testi di due autrici brasiliane, Christiana de Caldas Brito e Vera Lúcia de Oliveira, che scrivono in lingua italiana. Attraverso alcune delle loro opere più rappresentative si delinea una differente geografia identitaria animata dal movimento di separazione dalla terra madre.

PAROLE CHIAVE letteratura della migrazione; traduzione; identità.

RESLMO Este trabalho apresenta uma análise comparativa de alguns textos de duas autoras brasileiras, Christiana de Caldas Brito e Vera Lúcia de Oliveira, que escrevem em língua italiana. Mediante algumas de suas obras mais representativas se esboça uma diferente geografia da identidade, suscitada pelo movimento de separação da terra mãe. PALAVRAS-CHAVE literatura da migração; tradução; identidade.

ABSTRACT This paper brings a comparative analysis between texts by Brazilian authors Christiana de Caldas Brito and Vera Lúcia de Oliveira, who write in Italian. Some of their most representative work outline a different identity geography, brought about by the departure from the mother land.

KEYWORDS migration literature; translation; identity. 


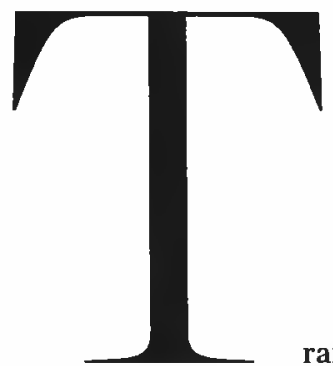

ransiti, attraversamenti, passaggi di tempo, paesaggi di parole, flussi di coscienza, migrazioni di metafora: ecco, scrivere è tutto questo. La scrittura può nascere da ciò che è già noto - dalla memoria del tempo, da un discorso antico, da idee ricevute - oppure percorrere confini, all'apparenza insormontabili, dove l'incontro con l'altro espone ad evenienze inedite e impreviste ${ }^{1}$

Nel panorama letterario italiano si trovano produzioni segnate dal limite della frontiera, i cui accenti diasporici si esprimono con una lingua che per gli autori - siano essi poeti, scrittori o saggisti - è definibile dislocata, in quanto differente dalla lingua delle loro origini. Alludiamo a una letteratura in lingua italiana nata da quella che il poeta e critico tedesco Hans Magnus Enzensberger definisce la "Grande Migrazione"2.

Fin dagli anni '90 del Novecento, Armando Gnisci segnalava l'originalità di questo fenomeno letterario ${ }^{3}$ che esprime insieme un ruolo semantico e politico di fuoriuscita dalla monofonia della lingua madre, per fondersi in una comunità polilinguistica e creolizzata. Una comunità in grado di procedere oltre il multiculturalismo acritico, per nutrirsi di nuove geografie identitarie con le quali raccontare storie di individui non più silenziosi, ma animati dal movimento che li separa dalla terra dei padri, dalla lingua e da tutto ciò che è differenza ontologica, in vista di nomadismi, trasversalità, ibridità ${ }^{4}$ Scrive Gnisci:

Gli scrittori migranti non appartengono a una, a due o a più nazioni, ma, per quelli della generazione di prima ondata che ha emigrato e ha scritto nella curva del transito, appartengono alla rete delle relazioni formata dalla migrazione transmondiale e a una nuova forma di cultura. [...] Questa rete, però, è visibile e apprezzabile solo

1. Si veda a questo proposito Chambers I., Paesaggi migratori, Costa \& Nolan, Genova, 1996, p. 15.

2. Citato da Armando Gnisci nel suo saggio introduttivo "Scrivere nella migrazione tra due secoli". In Gnisci A., Nuovo Planetario Italiano, Città Aperta, Troina, 2006, p. 13.

3. Gnisci A, Il rovescio del gioco, Crucci, Roma, 1992 ora incluso in Gnisci A, Creolizzare l'Europa. Letteratura e Migrazione, Meltemi, Roma, 2003.

4. Si veda a questo proposito Mariella Pandolfi e il suo lavoro dal titolo L'altro sguardo e il paradosso antropologico. Il suo saggio è introduttivo all'edizione italiana di Bhabha H., Nazione e Narrazione, Meltemi, Roma, 1997, pp. 10-25. 
nella lingua nazionale nella quale lo scrittore decide di costruire la casa del dopo, e quindi anche nella storia di quella lingua ${ }^{5}$.

Siamo in presenza di una letteratura che si definisce nei luoghi di transito di autori che, negli ultimi venticinque anni, hanno vissuto e vivono la condizione di migrante nella nostra penisola mediterranea. A questa nuova letteratura tradotta, come la definirebbe Salman Rushdie, appartengono, tra gli altri, scrittori di origine brasilian $a^{6}$, numerosi rispetto al resto degli autori provenienti dall'America Latina e composti nella maggioranza da donne e poeti. Un vero e proprio scambio culturale, quello tra Italia e Brasile, che vien fatto risalire al 1875, anno ufficiale dell'inizio della immigrazione italiana nella terra d'oltreoceano ${ }^{7}$. Al cuore del presente lavoro sono proprio due autrici brasiliane: Christiana de Caldas Brito, scrittrice, e Vera Lúcia de Oliveira, poeta.

Salman Rushdie ${ }^{8}$ riconosce all'emigrato un ruolo importante perché nel rinunciare alle radici, alla lingua e alle norme sociali di provenienza è stato costretto ad individuare nuove modalità espressive per descriversi. L'emigrazione stessa può essere, a suo avviso, definita per mezzo della figura retorica della metafora, la cui radice greca indica il "trasportare" delle idee in immagini" . Lo sradicamento linguistico diventa, dunque, uno degli aspetti più importanti della condizione di emigrato, che Rushdie rappresenta con la lumaca, la cui metafora esprime la lentezza el'incertezza derivanti dal destino - a tratti anche violento - di traduzione e di dislocazione. Tuttavia, per Vera Lúcia de Oliveira essa non necessariamente assume connotazioni negative. Scrive, infatti, la poetessa:

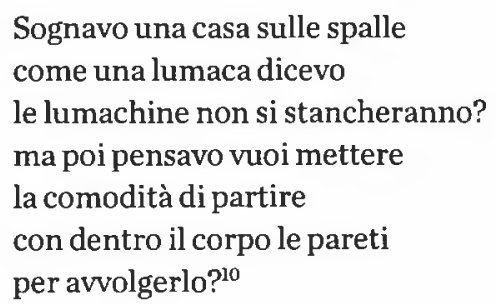

Ł́ la casa di poeta "che costantemente migra, sempre e comunque solo, "in due lingue" o in nessuna, perché forse non esistono poeti fuori dal silenzio dei cuori, come dice Fernando Pessoa"ll e come riporta Mia Lecomte.

5. Gnisci A., Creolizzare l'Europa, cit., p. 9.

6. Si consulti, a questo proposito, la banca dati on-line Basili (Banca dati degli scrittori immigrati che pubblicano in lingua italiana) attivata sul sito del Dipartimento di Italianistica e Spettacolo dell'Università "La Sapienza" di Roma: www.disp.let.uniroma.it/basili200l.

7. Tuttavia, prima di questa data, c’è traccia di una produzione letteraria in lingua italiana, perlopiù legata a scrittori brasiliani che, compiuti gli studi in Europa, amavano esibire la propria cultura o semplicemente seguire mode esterofile, tenuto conto che i loro lettori appartenevano alla corte imperiale. Un esempio da citare può essere Manoel Botelho De Oliveira, primo poeta brasiliano a pubblicare, nel 1705, un volume di poesie e madrigali in italiano, dal titolo Música do Parnaso. O ancora alcune opere di autori italiani con residenza temporanea in Brasile come Luis Vicente De Simoni e Galleano Ravara. Si rimanda per un approfondimento al saggio di Hohlfeldt A., "La letteratura di lingua italiana in Brasile". In Marchand J.-J. (a cura di), La letteratura dell'emigrazione. Gli scrittori di lingua italiana nel mondo, Fondazione Agnelli, Torino, 1991, pp. 205-212.

8. Rushdie S., "Günter Grass". In Rushdie S., Patrie immaginarie, Mondadori, Milano, 1991, pp. 297-306. A proposito dei personaggi migranti descritti da Salman Rushdie si consulti van Houtum H., van Naerssen T., Bordering, Ordering and Othering. In "Tijdschrift voor Economiche en Sociale Geografie", Blackwell Publishers, Oxford, Malden, vol. 93, n. 2, pp. 125-136. Gli autori scrivono: "In the Satanic Verses Rushdie (1988) pictured the immigrant as a person that is transformed in and by the strangers in the new and strange society as a strange creature, as if wearing horns, a tail and cloven hooves" (p. 132).

9. Ibidem, p. 303.

10. de Oliveira V. L., Verrà l'anno, Fara editore, Santarcangelo di Romagna (RN), 2005, p. 36.

11. Lecomte M., Nella culla di carta delle parole, in de Oliveira, V. L., Verrà l'anno, op. cit. p. 73. 
La letteratura di cui stiamo parlando è pura eversione che si oppone alla convinzione che è più semplice vivere relegati nella tradizione e nell'appartenenza, nella nostra casa, nella nostra nazione, nelle nostre narrazioni di nazioni considerate autentiche e pure. ̇̀ possibile, invece, collocarsi nell'"accanto", e inoltrarsi nella traduzione per dar vita a diverse forme di comunicazione capaci di superare l'afasia degli incontri mancati.

Non a caso, alcuni dei personaggi raccontati da Christiana de Caldas Brito vivono sospesi tra due mondi: uno perduto o alimentato solo nella memoria; l'altro per certi versi ancora ignoto, nel quale dovranno imparare a ridefinirsi e a tradursi. Questo significa collocarsi nel mezzo, lungo un confine privo di appartenenze ben delineate, in quello che Homi Bhabha definisce in-between space ${ }^{12}$, uno spazio intermedio, appunto, che si trasforma in luogo fecondo dove elaborare "le strategie del sé - come singoli o gruppo - che danno il via a nuovi segni di identità e luoghi innovativi in cui sviluppare la collaborazione e la contestazione nell'atto stesso in cui si definisce l'idea di società"13

Nella lettura di alcuni dei primi racconti di Christiana de Caldas Brito, è possibile vedere come la pratica di inserzione dell'italiano nella struttura linguistica preesistente si presenti come "interlingua", attuando un costante processo di traduzione in cui la lingua $B$, come la definisce la scrittrice nel racconto inserito nel nostro colloquio, vale a dire la lingua della memoria, è ancora presente. L'interlingua si è formata e continua a formarsi attraverso delle pratiche di imitazione, variazioni e calchí che la determinano come una lingua non stabilizzata. Nel racconto dal titolo Ana de Jesus possiamo vederne un esempio:

Bambina mia non mangia bene. Quando venuda in Italia, io ho comprado al supermercado latina di carne per mandare a mia figlia. Io pensava che ci stava foto di cagnolino bello per divertire i bambini. Poi, rideva molto io. Era carne per cane. Sorella mia scrive che inia figlia mai mangiado cosi buono. ${ }^{14}$

Oppure nel racconto Olinda:

Lui è abituato a sentire le parole che stanno zitte dietro le parole rumorose. Da poco tempo è riuscito a farmi il passaporte e penserà che era un plano mio per andare via. No, io espera che lui non poderà pensare questo, che solo non saberà spiegare il perché di mia partenza.

Di otro modo non posso fare. ${ }^{15}$

La de Caldas Brito ci ricorda che si tratta della stessa lingua che gli italiani emigrati in Brasile nell'Ottocento parlavano "mescolando" la lingua italiana con quella portoghese ${ }^{16}$. L'interlinguismo è stato definito una "lingua accanto alla lingua"17 e rappresenta la possibilità di creare un elemento

12. Bhabha H., Iluoghi della cultura, Meltemi, Roma, 2001. L'autore ritiene questo spazio "teoricamente innovativo" e "politicamente essenziale", in quanto interprete di un vivere inteso come arte del presente.

13. Ibidem, p. 12.

14. de Caldas Brito C., Amanda Olinda Azzurra e le altre, Lilith Edizioni, Roma, 1998, p. 30. Nella più recente edizione del 2004 è stato edito da Oédipus. Il racconto dal titolo Ana de Jesus, incluso in questa raccolta, è una versione rielaborata e ampliata di una precedente versione vincitrice dell'edizione 1994-1995 del premio Eks\&Tra. È stato anche rappresentato a teatro.

15. Ibidem, p. 83.
16. Si consulti a questo proposito l'articolo di Hohlfeldt A., La letteratura di lingua italiana in Brasile, op. cit. Oppure Franzina E., "Brasile: fra storia e romanzo", in Marchand J.-J. (a cura di), La letteratura dell'emigrazione. Gli scrittori di lingua italiana nel mondo, op. cit., pp. $213-228$. 17. Zaccaria P., La lingua che ospita, Meltemi, Roma, 2004, p. 145. 
completamente nuovo che nasce dalla combinazione di due che lo precedono diacronicamente in termini di storia e di cultura, ma che si inserisce all'interno di un sistema di comunicazione dove nulla viene inteso come errato ${ }^{18}$. Volendo ritornare a Homi Bhabha, l'interlingua è quel "terzo spazio" dove trova dimora l'ibridità. Scrive, a questo proposito, Antonio Perri:

[...] lo spazio ibrido è per definizione plastico, mobile, aperto a nuovi termini e nuovi linguaggi vi possono fare irruzione amalgamandosi gli uni con gli altri dinamicamente, senza mai imporsi come "il" modello - la frontiera unica, oltre la quale sta lo "straniero" 19

Questo spazio apre alla differenza, al dubbio, alla precarietà e alle insidie di mondi sconosciuti. Eppure, chi vive nella doppia condizione di migrante - dalla propria patria edalla propria lingua - percepisce prepotente l'assenza alla quale solo il movimento nomade delle parole riesce a dar voce.

Pois nenhuma resposta - scrive Mauro Maldonato - pode se dar, a não ser no olvido: o olvido que se torna ausência; a ausência que para dizer de si não necessita de raízes; raízes que o êxodo da língua materna e da ilusão de que uma palavra possa des-velar (e não apenas revelar) transforma em pura errância ${ }^{20}$.

È nello scritto, infatti, che si coagula quella originaria mancanza che tenta di prendere forma nella parola per raccontare, per rinnovare la fedeltà a se stesso e alla propria origine, trasfigurando nella lingua la liberazione da quei legami che permettono di infrangere le frontiere, anche quelle segnate dai limiti della pagina.

Può accadere, allora, di immergersi in un'atmosfera letteraria estraniante che rinvia ad un'esperienza di sradicamento, di non appartenenza, di non-assimilazione, cui è necessario far fronte, in un rapporto immediato e intimo con la differenza, con ciò che separa. Lasciamoci condurre dalla de Oliveira nei suoi Pezzi:

\author{
Sono frantumata \\ silenzi escono dalla bocca \\ tenui, \\ stavo disegnando \\ parole, \\ ho perso il modo di destarmi \\ sono in tanti pezzi \\ da essere quasi infinita ${ }^{21}$
}

\footnotetext{
18. Ibidem. La Zaccaria cita a questo proposito Bruce Novoa con riferimento ai suoi studi sugli autori chicanos, esempio rappresentativo della condizione di border culture e profondamente segnato da importanti questioni linguistiche. Si veda Novoa Bruce J., Chicano Authors: Inquiry by Interview, University of Texas Press, Austin, 1980.

19. Perri A, Nota del traduttore. In Bhabha H., Nazione e narrazione, op. cit., p. 30.

20. Maldonato M., Raizes errantes, SESC/Ed. 34, São Paulo, 2004, p. 31. Traduzione di Roberta Barni. Edizione italiana Maldonato M., Certe estremità della coscienza, Effatà Editrice, Cantalupa (To), 2005, p. 23.

21. de Oliveira V. L., Geografie d'ombra, Fonèma, Venezia, 1989.
} 
In questapoesia, nuove risonanze trovano eco nelle parole, inedite variazioni narrative definiscono la loro essenza. Il tempo esteriore del comunicare non sembra prevalere, mentre la parola, nel gioco della rappresentazione di sé, può raddoppiarsi o arrivare ad elidersi. Ė il crescere silenzioso di una lingua e di una immagine condivisa che, in una radicale differenza, annuncia nuove prossimità.

L'identità è simile a una ferita che rappresenta intera la sua fragilità, la sua incertezza, il suo dolore. Non può essere considerata un dato di fatto. Simile a una frontiera, da percorrere senza varcare, diventa un esercizio esistenziale e letterario che apre all'alterità. Anche Fernando Pessoa, poeta molto amato dalla de Oliveira, ha scritto di questa ferita.

Allora, è possibile comprendere che forse una vita non basta. Forse solo l'arte e la letteratura non sono sufficienti. Forse è necessario ascoltare i tanti "pezzi". O quei "cocci assurdamente consapevoli/Ma consapevoli di se-stessi/non degli altri cocci"22. Ascoltarli, dunque. E dargli voce. E dargli spazio. Scrive Fernando Pessoa:

Mi sono moltiplicato per sentirmi, per sentirmi ho dovuto sentire tutto, sono straripato, non ho fatto altro che traboccarmi [...]. ${ }^{23}$

Per Pessoa, che Eduardo Lourenço definisce "lo straniero assoluto", una patria esiste: è la lingua portoghese. Tuttavia, si coglie una inquietudine. Anche per chi ha trovato una nuova casa ${ }^{24}$, anche se "ora nella casa c'è posto per le rondini/se d'autunno non vogliono partire/ci sarà un letto caldo e cuscini/chiuderanno gli occhi/crederanno di volare ancora/godendo il tepore del sole"25.

$\grave{E}$ importante, dunque, auto-rappresentarsi per diventare una figura visibile agli occhi di chi ci percepisce come straniero, ma altresì per "sentirsi". Occorre raccontarsi e per farlo si può ricorrere alle due diverse lingue che appartengono alla storia di chi narra: l'italiano e il portoghese. Questa volta, però, non utilizzando il "portuliano", ma consentendo l'emersione della lingua della memoria, "la lingua materna con il suo latte" ${ }^{26}$ che riporta ricordi dolce-amari e si fa largo tra la lingua dell'accoglienza. Ciò in considerazione del fatto che nello spazio interstiziale tra due lingue e due culture è possibile la "non scelta", anche se inconsapevole, che conduce a una sperimentazione semiotica e culturale.

Christiana de Caldas Brito nel suo racconto Jose $e^{27}$ compie una "non scelta", o meglio - come sostiene la scrittrice - forse è il suo personaggio e le sue voci che l'assediano a lasciar spazio ad entrambe le lingue:

Si alza dalla brandina per accendere la piccola stufa. Gli fanno male le ossa. Forse per l'umidità, forse per i lavori pesanti degli ultimi giorni.

"Permesso?"

Entrano tutti.

22. Pessoa F., Fantasie di interludio. Antologia di Pessoa. I Pessoa di Pessoa, a cura di F. Cabral Martins. Passigli editori, Firenze-Antella, 2002, p. 156.

23. "Passaggio delle ore" in Pessoa, F. Poesie di Álvaro de Campos, a cura di M.J. de Lancastre, Traduzione di Antonio Tabucchi, Milano, Adelphi, 1993, $5^{\circ} \mathrm{ed}$.

24. Djebar A., Queste voci che mi assediano, II Saggiatore, Milano, 2004, p. 183.

25. de Oliveira V. L., Verrà l'anno, op. cit., p. 29.

26. Djebar A., Queste voci che mi assediano, op. cit., p. 33.

27. de Caldas Brito C., Qui e là, Cosmo Iannone Editore, Isernia, 2004, pp. 109-112. 


\section{“Parabéns pra você, nesta data querida, muitas felicidades, muitos anos de vida's \\ Come mai cantano nella sua lingua ${ }^{28} ?$}

Queste dissonanze interne - che si colgono nelle architetture narrative e nei paesaggi letterari della loro diglossia, ma anche delle altre lingue europee con le quali queste autrici entrano in contatto - aprono il proprio spazio e il proprio orizzonte all'erranza, come momento di commiato dalla propria terra e dal proprio linguaggio.

Siamo di fronte a una scrittura orizzontale che non ci sollecita verso la fine della narrazione o della poesia, ma ci chiede attraversamenti a volte dolorosi. Sfiorare il "fuori campo" delle due lingue e la loro sostanza è entrare in una zona nevralgica, nel cuore di una lingua pronta a tradursi, anche se non sempre riesce a farsi comprendere. Ma diamo voce a Vera Lúcia de Oliveira:
ora in due lingue da sola
parlo non so a chi il vento
capisce pare e porta via
al mittente il messaggio ${ }^{29}$

Fra due lingue, dunque. Come Tempo de doer/Tempo di soffrire $e^{30}$, raccolta di poesie bilingue anche nel titolo, perché, come scrive la poetessa in una sua nota,

Mentre le poesie sgorgavano, andavo da una lingua all'altra e mi pareva che certe cose le potessi dire solo nel mio portoghese materno, intimo, viscerale, o solo nel mio italiano intrinseco, quotidiano, familiare: entrambe le lingue come porzioni di anima, di pelle, di carne e di cuore. ${ }^{31}$

Ora, se la traduzione - come sostiene la de Oliveira - è "un avvicinamento al testo e all'altro che esprime ed è espresso dal testo", tradurre è una forma di conoscenza, ma anche di lettura che rende possibile un dialogo col testo, dove il traduttore-lettore trova il proprio spazio, per certi versi circolare, tra l'originale e la traduzione che ne deriva ${ }^{32}$.

Per una letteratura che si nutre del margine, quasi sospesa come un ponte, è inevitabile porsi un quesito in merito alla possibilità di affacciarsi sul "testo in traduzione" fino ad aprire la propria lingua ad altre sensibilità. 
In questa accezione - scrive Alessandro Corio - il termine traduzione, andando ben oltre il contesto linguistico-letterario nel quale è solitamente impiegato, diventa sinonimo di transculturazione, [...] che consente di trasportare da un luogo all'altro non solo parole, ma anche concetti, idee, costumi, religioni, immagini e simboli [.... ${ }^{33}$

Ma tutto questo rifiutando una traduzione culturale dove l'altro, marginalizzato, viene tradotto, appreso e descritto attraverso il ricorso a termini familiari, che non prescindono da una impostazione eurocentrica pronta a ridurre l'autore, originario di un altro paese, a un semplice informatore della cultura di provenienza ${ }^{34}$. In quest'ultimo caso, a una specie di Caliban che "impara il prosperese e fa la spia contro se stesso"35.

Negli esempi delle nostre autrici, invece, la traduzione è "uno dei modi più radicali per cercare di conoscere l'Altro" ed esprimere il desiderio di dialogare, di incontrare la differenza senza la violenza del ricondurre a sé, ma di percorrere anche l'ambiguità insita nella traduzione, quella che la de Oliveira definisce "la ricerca di una identità comune". Naturalmente, nella consapevolezza che l'incontro introduce a una inevitabile separazione perché

Dificil - scrive Maldonato - um encontro que se entrega a uma separação, à intangibilidade de nomes e signos, ao xeque que as palavras padecem ao sobreviver ao silêncio e ao vazio que elas deixam ao desaparecer, como o fogo as cinzas. ${ }^{36}$

Ora se la casa, come abbiamo detto in precedenza, ha valore di ponte; se la parola nasce e si nutre del confine; se prepotente è la sensazione dell'impossibilità di un ritorno a casa; condurre l'altro oltre il ponte è mostrargii l'importanza di un avvicinamento, ma, sopra ogni cosa, che non tutto è traducibile, fino a fargli scoprire che in un nuovo incontro non sempre sarà possibile parlare la stessa lingua.

Le nostre traduttrici-autrici, infatti, redigono testi bilingui che si autotraducono all'interno di uno stesso testo o passano da una lingua all'altra, introducendo delle tecniche narrative e poetiche di grande interesse.

L'esempio può essere dato dal primo romanzo di Christiana de Caldas Brito, dal titolo 500 temporali, con il quale la scrittrice torna a Rio de Janeiro, sua città natale, per condurci lungo le strade malfatte di un morro: quello di Santa Teresa, alle pendici del monte Corcovado ${ }^{37}$

Quella discontinuità comunicativa, che in precedenza avevamo avuto modo di riscontrare, in questo romanzo si trasforma, fino a consentire l'individuazione di aree problematiche che, da un punto di vista linguistico, possono essere fatte rientrare nel doppiaggio cinematografico. Si, perché ci troviamo di fronte ad una scelta linguistica molto simile a quella della traduzione al cinema.

Come è possibile lasciar emergere dall'anima italiana termini portoghesi legati a espressioni fisse (tudo bem?), a frasi idiomatiche (Nossa Senhora Aparecida!), a proverbi, a riferimenti

33. Corio A. "Elogio dell'ibridità, esotismo postcoloniale e mercato globale: per una genealogia critica del discorso della contaminazione", Experience, 10, 2006, Edizioni Sperimentali, Mattioli 1885 S.p.A., pp. 9-21.

34. Spivak G. C., Critica della ragione postcoloniale, Meltemi, Roma, 2004.

35. Si veda a questo proposito il saggio di Wa Thiong'o N., "L’Africa e i suoi interpreti, spie dello straniero o pionieri della libertà". In Gnisci A., Poetiche africane, Meltemi, Roma, 2002,pp. 65-78.

36. Maldonato M., Raizes errantes, op. cit., p. 31.

37. Si veda Di Lucchio P., 500 anni di temporali. Recensione al primo romanzo di de Caldas Brito C., 500 Temporali. Cosmo Iannone Editore, Isernia, 2006. Kuma, n. 2/2006,www. disp.let.uniromal.it/kuma/kumahtml 
urbanistici o geografici? Oppure come tradurre la parola scritta su cartelli o titoli di giornali (é proibido fumar; Liberdade ainda que tardia)? E ancora, come occuparsi della resa linguistica di canzoni, filastrocche, racconti (Dorme, meu filhinho,/fica quieto pra dormir/senão bicho papão/vai levar você daqui)? Delle risposte a questi interrogativi possono trovarsi nell'analisi di un rapporto dialogico tra le lingue che abitano lo scrittore/il testo, fino a creare tra loro delle trattative continue ${ }^{38}$. In questo "doppiaggio" letterario le frasi, le parole, le espressioni non possono, e non vogliono, essere tradotte per una necessità di fedeltà linguistica alla versione originale. Una sorta di trompe-l'oreille la cui origine, oltre al rispetto della verosimiglianza, è dato dalla compresenza delle due lingue nella scrittrice.

Il testo di 500 temporali è disseminato di espressioni in portoghese che, come in un film tradotto, ci ricordano che la storia è ambientata in Brasile. E non solo quella del romanzo.

Ma può invece accadere, come nel caso di Vera Lúcia de Oliveira, che la lingua portoghese occupi uno spazio cosi vasto da non lasciare posto ad altro. Nella raccolta di poesie No coração da boca/Nel cuore della parola la de Oliveira ha dovuto affidare la traduzione in italiano a Guia Boni perché, come lei stessa afferma nel nostro colloquio, "tutto il mio essere si rifiutava di lasciare lo spazio che ancora abitavo dopo averlo scritto"

Questo perché non sempre ciò che si scrive può essere circoscrittoe perché, inoltre, può accadere che una delle due lingue può offrire per prima le sue parole. Parole, in quel momento, intraducibili all'animo del poeta. Appartengono ai ricordi, a una terra ormai lontana, a una immemorabile memoria talvolta dimenticata. Allora, la lingua seppellita prende il sopravvento, smette di essere doppia per dire l'opacità del mondo, per dire ciò che forse non si sarebbe voluto dire.

Édouard Glissant considera la traduzione una delle più importanti artidel futuro perché riserva a tutte le lingue, nessuna esclusa, una sovranità in quel momento assoluta. È un linguaggio, quello a cui dà vita il traduttore, capace di produrre l'imprevedibile, dove l'immaginario e la lingua danno corso a una vera e propria creolizzazione. Perché come scrive Glissant:

La traduzione, arte dello sfiorarsi e dell'avvicinarsi, è una pratica della traccia. [...] Tracciare nelle lingue è tracciare nell'imprevedibile della nostra condizione ormai comune. ${ }^{39}$

L'analisi di autrici cosi diverse come Christiana de Caldas Brito e Vera Lúcia de Oliveira, che in comune possiedono una patria, sia d'origine sia d'elezione, aiuta a comprendere l'importanza che la lingua e la traduzione rivestono in una letteratura come quella che in Italia viene definita della migrazione.

Nell'introdursi nelle loro opere, il lettore ha modo di percorrere quel ponte, senza attraversarlo, per ascoltare tutte le lingue del mondo. "Ma scrivere - come sostiene Glissant - in presenza di tutte le lingue del mondo non vuol dire conoscere tutte le lingue del mondo. [...] Vuol dire che 
la mia lingua la dirotto e la sovverto non operando attraverso sintesi, ma attraverso aperture linguistiche che mi permettono di pensare i rapporti delle lingue tra loro, oggi, sulla terra [...]"40

Tutto ciò, nella consapevolezza che la scelta di una vita e di una poetica vissuta sul confine può essere difficile, ma alleggerita dalla vana promessa di un ritorno:

\author{
per certi bordi cammino mamma \\ ma guardo bene non ti inquietare \\ so stare attenta e quasi scivolo \\ ma poi ritrovo l'equilibrio \\ se non dovessi più farcela \\ prometto che ti richiamo. ${ }^{41}$
}

\title{
Bibliografia:
}

Bhabha H., I luoghi della cultura, Meltemi, Roma, 2001.

Bhabha H., Nazione e Narrazione, Meltemi, Roma, 1997.

Bregola D., Il catalogo delle voci. Colloqui con poeti migranti, Cosmo Iannone Editore, Isernia, 2005.

Calvino I., Saggi 1945-1985, Arnaldo Mondadori Editore, Milano, 1995.

Chambers I., Paesaggi migratori, Costa \& Nolan, Genova, 1996.

Corio A., Elogio dell'ibridità, esotismo postcoloniale e mercato globale: per una genealogia critica del discorso della contaminazione, “Experience”, 10, 2006, Edizioni Sperimentali, Mattioli 1885 S.p.A.

de Caldas Brito C., Amanda Olinda Azzurra e le altre, Lilith Edizioni, Roma, 1998.

de Caldas Brito C., Qui e là. Cosmo Iannone Editore, Isernia, 2004.

de Caldas Brito C., 500 temporali, Cosmo Iannone Editore, Isernia, 2006.

de Oliveira V. L., Geografie d'ombra, Fonèma, Venezia, 1989.

de Oliveira V. L., Tempo de doer/Tempo di soffrire, Pellicani, Roma, 1998.

de Oliveira V. L., Verrà l’anno, Fara editore, Santarcangelo di Romagna (RN), 2005.

de Oliveira V. L., O lugar e a língua. "Revista de Italianística", 14, Universidade de São Paulo, 2006, pp. $97-114$.

Derrida J., De la grammatologie, Minuit, Paris, 1967.

Di Fortunato E., Paolinelli M., La questione del doppiaggio, Aidac, Roma, 1996.

Di Lucchio P., 500 anni di temporali. Recensione al primo romanzo di de Caldas Brito C., 500 Temporali. Cosmo Iannone Editore, Isernia, 2006. "Kuma", n. 2/2006, www.disp.let.uniroma.it/kuma/kumahtml

Djebar A., Queste voci che mi assediano, Il Saggiatore, Milano, 2004.

Glissant É., Poetica del diverso, Meltemi, Roma, 1998.

Gnisci A. (a cura di), Introduzione alla letteratura comparata, Bruno Mondadori, Milano, 1999.

Gnisci A., Poetiche africane, Meltemi, Roma, 2002.

Gnisci A., Creolizzare l'Europa. Letteratura e Migrazione, Meltemi, Roma, 2003.

Gnisci A. (a cura di), Nuovo Planetario Italiano, Città Aperta, Troina, 2006.

Khatibi A., L'amore bilingue, Edizioni Lavoro, Roma, 1993.

40. Ibidem, p. 33.

41. de Oliveira V. L., Verrà l'anno, op, cit., p. 43. 
Kristof A., L'analfabeta. Racconto autobiografico, Edizione Casagrande, Bellinzona, 2005. Maldonato M., Raizes errantes, SESC, Ed. 34, Sāo Paulo, 2004.

Marchand J.-J. (a cura di), La letteratura dell'emigrazione. Gli scrittori di lingua italiana nel mondo, Fondazione Agnelli, Torino, 1991.

Morin E., Il metodo 5. L'identità umana, Raffaello Cortina Editore, Milano, 2002.

Pessoa F., Fantasie di interludio. Antologia di Pessoa. Il Pessoa di Pessoa, Passigli editori, Firenze-Antella, 2002.

Rushdie S., Patrie immaginarie, Mondadori, Milano, 1991.

Spivak G.C., Critica della ragione postcoloniale, Meltemi, Roma, 2004.

van Houtum H., van Naerssen T., Bordering, Ordering and Othering. In "Tijdschrift voor Economiche en Sociale

Geografie", Blackwell Publishers, Oxford, Malden, vol. 93 n. 2, pp. 125-136.

Zaccaria P., La lingua che ospita, Meltemi, Roma, 2004. 


\section{APPENDICE}

\section{Come un viaggio nell'anima}

\section{Colloquio con Vera Lúcia de Oliveira}

Vera Lúcia de Oliveira, nata a Cândido Mota in Brasile, vive in Italia dal 1983. Ricercatore presso la Facoltà di Lingue e Letterature Straniere dell'Università di Lecce, alterna l'attività di ricerca con la traduzione e la composizione poetica. La sua raccolta Verrà l'anno ha vinto, in Italia, il premio "Popoli in cammino 2005"

1. António Lobo Antunes, nel suo ultimo libro-epistolario che raccoglie le lettere inviate dall'autore alla moglie, scrive: "91 giorni/91 giorni per poterti toccare [...]/La mia saudade è infinita e dolorosissima". In questo passaggio, a mio avviso, sono contenuti alcuni elementi che definiscono la saudade: il tempo, la lontananza, l'attesa. La saudade in Brasile è un sentimento "altro": una nostalgia anticipata o malinconia dell'assenza. In un discorso di ricezione letteraria, quanto di questa parola-sentimento entra nella traduzione?

Si è tanto parlato della difficoltà di tradurre questa parola che definisce un sentimento molto complesso e contraddittorio. Mi hanno chiesto giustamente di fornire alcuni elementi di questa complessità, cosa che ho cercato di fare in un saggio: "L'ambivalenza del termine, intuita già nei testi più antichi, si è conservata nelle definizioni moderne e il Novo Aurélio lo caratterizza come "il ricordo nostalgico e, allo stesso tempo, soave, di persone o cose distanti o estinte, accompagnate dal desiderio di tornare a vederle o ad averle"42 Concetto diverso da quello correlato alla "nostalgia" italiana, che indica "uno stato d'animo corrispondente al desiderio pungente o al rimpianto malinconico di quanto è trascorso o lontano"43

Si può provare saudade per una terra, una patria, una casa, una persona cara; si può provare saudade del grembo materno e dell'infanzia, di un passato gioioso perduto, di un mito, di un eroe, di un sogno, di un ideale, di un eden, di un messia. Nella fenomenologia della saudade, l'ambiente fisico 
è spesso determinante e intensifica o attenua sentimenti ed emozioni correlate, quali malinconia, uggia, ansia, trepidazione, tenerezza, attesa, gioia, dolcezza. [...] Per la connotazione ontologica del termine, il poeta portoghese Teixeira de Pascoaes farà di tale sentimento singolare, all'inizio del secolo XX, addirittura una religione, una poetica e una filosofia, il Saudosismo. [...]

Che la saudade possa definire un'identità non pare inverosimile, sempre che la si intenda come multipla e composita, giacché essa, oltre ad essere un sentimento, è un modo di vivere, di pensare e di sentire. Ė un legame con il passato o un recupero della memoria - del singolo o della comunità - che si proietta nel presente e nel futuro; una nostalgia non della felicità avuta e perduta, ma una nostalgia di essere felici ancora, anzi, una speranza di esserlo. In questo senso, la saudade è forse uno dei sentimenti più ardenti e utopici, è un ponte fra persone, è un legame fra luoghi diversi che convivono nella coscienza e nell'anima.

Le parole nascono legate ad una geografia e ad una storia. La saudade è legata alla storia portoghese, ma anche a quella brasiliana, entrambi paesi di gente sradicata, di gente che ha nostalgia di una casa, o di ricostruire la propria casa, che sarà sempre diversa, sarà altra, perché non ci sono due lingue uguali né due case uguali. La saudade è un sentimento di straniamentoe in questo senso ha molto a che fare con la traduzione. Anche la traduzione è cercare la propria casa in un'altra lingua, cosa impossibile in partenza, come affermano Croce, Ortega y Gasset e tanti altri studiosi. Eppure proviamo sempre a tradurre e la traduzione è un processo di riavvicinamento a questa casa perduta.

2. "Quanti sono gli io? Chi sono io? Cos'è questo intervallo che c'è tra me e me?". Fernando Pessoa, poeta da lei amato, e i suoi eteronomi ci conducono in una dimensione estranea al "principio di non contraddizione" che riguarda ogni discorso sull'identità. La desoggettivazione, che Pessoa compie per allontanarsi dalla propria esistenza, mi rimanda a quell'essere "altri" che è possibile sperimentare negli attraversamenti linguistici. Mi può parlare di come vive lei il rapporto tra identità e traduzione?

Se la traduzione è un avvicinamento al testo e all'altro che esprime ed è espresso dal testo, allora la traduzione è un processo anche di empatia, è un cercare di entrare nell'ídentità dell'altro. Il rapporto che si instaura non può che essere empatico e dunque l'io cerca di sentire tramite l'altro, di vedere con gli occhi dell'altro il mondo, o quella parte di mondo che si guarda. L'io non sarà mai completamente l'altro, perché l'unico modo di spersonalizzarsi o "desoggettivazionarsi" completamente è nella malattia, nella schizofrenia, o nella morte. E, tuttavia, non c'è altro modo di avvicinarsi agli altri se non cercando di uscire da noi e provando ad "entrare" nelle ragioni, nell'essenza e nell'identità di chi è diverso da noi. Tradurre è conoscere, è forse uno dei modi più radicali di cercare di conoscere l'altro che ci attrae, ma che è irriducibilmente distinto e dissimile da noi. 
3. Italo Calvino, nel suo saggio Sul tradurre, afferma che un testo tradotto è un miracolo che avviene grazie al traduttore letterario capace di mettere "tutto se stesso in gioco per tradurre l'intraducibile". So che lei alla sua attività di poetessa e di ricercatrice universitaria affianca quella di traduttrice. Come avviene la sua scelta delle opere da tradurre? C'è una analogia tra la sua poetica di autrice e quella di traduttrice?

Manuel Bandeira ha affermato che traduceva solo quello che avrebbe voluto scrivere. Per me è la stessa cosa. Traduco i poeti che mi attraggono, che mi affascinano e che hanno affinità con me. Non potrei tradurre autori che non amo, che sento troppo distanti, per modo di essere e di pensare. Per quanto possa sembrare diverso un autore, quando scelgo di tradurlo è perché ho identificato in lui qualcosa di intrinseco anche a me stessa. In realtà, l'ambiguità della traduzione sta tutta qui: si pensa di uscire per cercare l'altro e forse, in realtà, si cerca nell'altro quella parte di condivisione che abbiamo con lui, quella parte di identità comune. Che dialogo ci potrebbe mai essere fra due che non hanno nessun tipo di affinità o di reciprocità?

4. Assja Djebar definisce la lingua francese "lingua matrigna" " "avversa" in quanto lingua che dice l'avversità. Per Assja Djebar il francese è, però, la lingua del colonizzatore. In una sua intervista, lei dichiara che a un certo punto ha temuto di perdere la sua lingua materna, il portoghese. È possibile, secondo lei, trasformare il bilinguismo in una ricchezza culturale da utilizzare in chiave neutra?

Nel mio caso sì, è possibile. Non ho un rapporto conflittuale con l'italiano. Non era per me la lingua del colonizzatore. Neanche il portoghese lo è, perché è una lingua acquisita in Brasile tanto tempo fa, ha avuto tutto il tempo per con-formarsi alla cultura brasiliana come una pelle sul corpo, come un vestito sulla pelle. Dunque, ho due lingue che per me sono lingue che accolgono, sono materne nel senso vero del termine, di rapporto che ricorda il legame di affetto (e di feto) con la madre. Solo che il portoghese è arrivato prima, l'italiano dopo. Proprio per vivere in questo modo il rapporto con l'italiano, cioè senza animosità, senza avversarlo dentro di me, ho pensato che avrei potuto correre il rischio di perdere il rapporto privilegiato con il portoghese come lingua poetica. Poi, ho capito, vivendo e sentendo le due lingue dentro di me, che questo non poteva succedere, non correvo tale rischio. Le due lingue sono padrone di casa, come due madri che attendono il figlio, due madri che lo hanno partorito miracolosamente. Solo che una lo ha partorito prima e gode di questa priorità. L'altra lo accetta.

5. La radicalizzazione del bilinguismo è, secondo Abdelkábir Khatibi, un "rêve fou", al punto che l'unica via di fuga da questo sogno folle può trovare compimento solo nella scrittura e nella cultura. $\dot{E}$ d'accordo con lo scrittore maghrebino? 
Non vedo il bilinguismo come un sogno folle, ma come un viaggio nell'anima. C'erano parti di me stessa a cui non avevo accesso in portoghese. Scrigni chiusi al mio occhio folle (questo si folle, perché vuole guardare ovunque). C'erano porte sbarrate che non aprivo con la mia prima lingua. È stato necessario acquisire un altro idioma, con la stessa intimità con cui ho acquisito l'italiano, per poter scandagliare tutta intera la mia anima (e forse non ancora tutta, chissà se avrò bisogno di un'altra o di altre lingue ancora...). Il fatto è che quello che scrivo in portoghese, l'ho anche visto e sentito in portoghese, e lo stesso avviene per l'italiano.

6. Nel No coração da boca/Nel cuore della parola ho notato che ha affidato la traduzione a Guia Boni. La scelta di non occuparsi della traduzione in prima persona è legata al fatto che tradurre in un'altra lingua è per l'autore una riscrittura capace di modificare l'assetto originario dell'opera?

Ho scelto allora, quando è stato pubblicato, di non tradurre questo libro perché lo avevo scritto da poco e tutto il mio essere si rifiutava di lasciare lo spazio che ancora abitavo dopo averlo scritto. Non riuscivo a passare cosi presto all'altra lingua, nel processo di traduzione di un testo scritto da poco. Per me una cosa è la scrittura, un'altra la traduzione. La scrittura ha a che fare con un'epifania. Quando mi metto a scrivere un libro, il libro è già scritto dentro di me, l'ho elaborato lentamente, giorno per giorno, senza fretta. Quando è pronto, debbo scriverlo, se non lo faccio sento di perderlo per sempre.

Invece tradurre è un lavoro analitico. È necessaria la ragione, accanto alla sensibilità e alla capacità di inventare forme e parole. Tuttavia, non è lo stesso tipo di creazione della poesia in sé, nel suo momento di rivelazione, in cui c'è qualcosa che sfugge alla logica e alla ragione, oppure qualcosa che appartiene ad una logica diversa, che attraversa altri sensi e altri canali per farsi parola. Alcuni studiosi dicono che tradurre e creare un testo poetico sia la stessa cosa. Per me non è lo stesso, per questo ho bisogno di tempo, fra il momento di rivelazione della parola e il momento di analisi di questa parola. Riesco ad auto-tradurmi solo dopo parecchio tempo che ho scritto un libro.

E poi mi piace vivere l'avventura dell'altro quando traduco, l'avventura che mi porta ad avvicinarmi all'altro o agli altri. Proprio per questo ultimamente non mi piace auto-tradurmi. Alcuni pensano che questo sia un atteggiamento, ma non lo è. Forse è un sentire legato a questo momento, non posso dirlo. 


\section{Lingua $B$.}

\section{Scrivere nella lingua dell'altro}

\section{Colloquio con Christiana de Caldas Brito}

Christiana de Caldas Brito, nata a Rio de Janeiro, vive in Italia da moltianni. Laureata in Psicologia all'Università "La Sapienza" di Roma e all'Università di San Paolo del Brasile, è, inoltre, diplomata presso la Scuola di Arte Drammatica (Università di San Paolo). Ė psicoterapeuta, scrittrice di racconti, romanzi e testi teatrali.

1. "La lingua vive come un grande albero le cui radici sono nel sottosuolo della vita sociale e della vita cerebrale, le cui fronde si sviluppano nel cielo delle idee e dei miti, ele cui foglie frusciano in miriadi di conversazioni". Edgar Morin, attraversatore di lingue, cosi parla de L'umanità del linguaggio nel suo libro L'identità umana. Come si presenta il "grande albero" di una scrittrice brasiliana che ha scelto la lingua italiana per esprimersi?

Prima di costituirsi in parole, una lingua è fatta di elementi visivi, di colori, di odori e suoni, di sensazioni e ricordi. È quello a cui, penso, si riferisca E. Morin quando parla di "sottosuolo della vita sociale e della vita cerebrale". A poco a poco, con l'abitudine, associamo gli stimoli ricevuti dai sensi a dei suoni precisi (le parole) che finiscono per acquisire un significato. La lingua di origine è una forma mentis, una specie di fodera dell'anima. Una lingua che si impara da adulti è come una casa nuova. L'adattamento al nuovo territorio costa fatica. Ci vuole un po' di tempo finché possiamo dire della nuova lingua, come della nuova casa, "la sento come mia".

Le trascrivo (sotto) un testo che ho scritto sulle parole di una migrante. Il testo si chiama Linea $B$ e si riferisce ad una delle linee della metropolitana di Roma. Potrebbe anche chiamarsi "Lingua B", una specie di lingua usata dai migranti e che all'inizio è come una lingua di serie B. Solo quando ci sentiamo affettivamente bene con le parole, riusciamo ad essere creativi in una nuova lingua.

2. Nella suaprima raccolta di racconti, Amanda Olinda Azzurra e le altre, alcuni dei suoi personaggi parlano in "portuliano", lingua che nasce dalla combinazione delle sue due lingue: il portoghese e l'italiano. "Il risultato - la cito riportando un brano della sua postfazione al libro - è una voluta 'sgrammatizzazione' della lingua italian a che riflettela loro mentelusofonica". Sonorimasta colpita dalla "mente lusofonica". Può provare a restituirci immagini più dettagliate? 
Quando gli emigrati italiani arrivarono in Brasile, alla fine dell'Ottocento, si sforzavano di parlare il portoghese. In realtà, mescolavano le due lingue (l'italiano e il portoghese), creando una lingua ibrida, il "portuliano". Oggi, in Italia, si verifica il fenomeno inverso: i brasiliani venuti in Italia si sforzano di parlare l'italiano ma in realtà la loro mente lusofonica pensa in portoghese ed effettua un sommario passaggio ("traduzione") nella lingua di Dante.

3.Agota Kristof, scrittrice ungherese che scrive in francese, ha definito nel suo libro L'analfabeta. Racconto autobiografico la lingua francese una lingua nemica. Questo non solo per la difficoltà che incontra nel parlarla e nello scriverla, ma anche perché sta uccidendo la sua lingua materna. Invece, lei nel suo incontro con la lingua italiana sembra divertirsi. In Qui e là, sua ultima raccolta di racconti, alcuni di questi come Ià la vecchiaia, Il volo, Tutti sono dei veri epropri divertissement linguistici. Quanto si riflette su questa giocosità il ritmo portoghese?

Il mio amore per le parole si esprime prima di tutto in un aspetto ludico che porto dall'infanzia. Mia madre si divertiva con noi (mia sorella, mio fratello ed io), inventando delle parole, trovando delle rime ai versi, cambiando gli aggettivi presenti nei testi delle favole e così via. Questo mi ha dato il coraggio di osare con le parole anche in italiano. In quanto straniera, vedo la vostra lingua dal di fuori. Il mio punto di vista è diverso da quello di una italiana che ha formato la sua mente con la lingua italiana. Per me, i vostri vocaboli non hanno una storia, sono vergini di ricordi, invitano all'esplorazione. É divertente condensarli, separarli, fonderli e confonderli. La lingua italiana offre alla mia esplorazione una serie di suoni che mi intrigano. Non la sento affatto come nemica.

4.Italo Calvino, nel suo saggio Sul tradurre, afferma che un testo tradotto è un miracolo che avviene grazie al traduttore letterario capace di mettere "tutto se stesso in gioco per tradurre l'intraducibile". Quella della traduzione è una operazione che compie anche una scrittrice come lei, in bilico tra due lingue? Si può parlare di una letteratura auto-tradotta?

$\mathrm{Si}$, spesso succede il fenomeno dell'auto-traduzione, ma più che altro con alcune parole. Spesso le parole si presentano in portoghese (la mia mente lusofonica...). Quando capita, non perdo il flusso creativo. Lascio la parola come viene e la sostituisco dopo. La sostituzione dipende non solo dal senso ma soprattutto dal ritmo.

5.Nel suo primo romanzo, 500 temporali, lei ritorna in Brasile. A Rio de Janeiro sua città natale. In questo suo ultimo lavoro due sono gli elementi, a mio avviso, che ne consentono una differenziazione rispetto ai precedenti. Il primo è una cifra stilistica dove la lingua italiana si affianca a quella portoghese. Quest'ultima compare in versi, frasi, storie che appartengono alla tradizione orale portoghese. Il secondo elemento è legato a una cifra intimistica che ha per compagna la saudade che mai, noi lettori, 
avevamo incontrato con queste tonalità. Cosa è cambiato stilisticamente in Christiana narratrice? Consideriamo archiviata Ana de Jesus, uno dei suoi primissimi personaggi che parlava in "portuliano"?

Pierangela, quando scrivo, non mi pongo certe domande. Non mi chiedo per esempio: qual è la mia attuale cifra stilistica e in che modo si differenzia dalle cifre precedenti? Quando scrivo, io voglio semplicemente raccontare una storia. Sono i lettori e i critici che dovranno porsi certi problemi. In realtà, sta a Lei dirmi che cosa è cambiato in me come narratrice. Io cambio perché sono un essere vivo, aperto alla realtà. Ana de Jesus più che un personaggio è uno dei tanti aspetti della mia scrittura. Tornerà? Ė archiviata? Non lo so. Una domanda pirandelliana: sono gli scrittori che creano i loro personaggi o sono i personaggi a creare lo scrittore?

6.Per concludere, so che sta lavorando al suo ultimo romanzo. Questa volta ambientato in Italia. La lingua portoghese farà ancora irruzione?

La risposta precedente serve anche a questa domanda. A dire il vero, non lo so se la lingua portoghese farà irruzione nel mio romanzo futuro. L'unica cosa che so è che scrivo lentamente e quasi sempre finisco per percorrere strade ignote alla partenza. 


\section{Linea B}

Le ho seppellite tutte. E adesso ho un cimitero di parole dentro. Sono vuote di ricordi le parole che uso al lavoro, alla metro, negli incontri per la strada. Parlo con la gente, senza toccare le parole di dentro. "Eur Palasport, prossima fermata Magliana. Eur Palasport, next stop Magliana." Che fare con le parole sepolte che non riesco a dimenticare, che di notte cantano come cicale, parole impazzite che odorano di mango? Per tutti questi anni le ho mantenute chiuse, come i dolcetti di goiaba avvolti nella pannocchia del mais.

"Magliana, prossima fermata Marconi. Magliana, next stop Marconi."

La porta della metro si apre. Insieme alle persone, entra il vento di tramontana. Sulle mie parole però continua a soffiare lo scirocco.

"Marconi, prossima fermata San Paolo Basilica. Marconi, next stop San Paolo Basilica." In quale regione di me si nasconde quello che penso e non so dire? La lingua natale è una barca che porta i pensieri. Spinti dalla corrente, i pensieri arrivano senza sforzo all'altro lato del fiume.

"San Paolo Basilica, prossima fermata Garbatella. San Paolo Basilica, next stop Garbatella." Le parole di dentro mi assalgono come lampi, accordi musicali improvvisi, un qualcosa che scatta.

"Garbatella, prossima fermata Piramide. Garbatella, next stop Piramide."

Lontano dalla foresta, il leone non può ruggire come prima. Le parole che ho lasciato erano per me lunghe braccia con cui toccavo l'orizzonte.

"Piramide, prossima fermata Circo Massimo. Piramide, next stop Circo Massimo."

Una signora si alza. Mi siedo. Il vagone della metro è una bocca chiusa. Fortunatamente entra un suonatore. Parla con le mani, lui. I suoni della sua fisarmonica danno ritmo alle vecchie parole che ballano nel mio petto come in un salone illuminato. Sorrido.

"Circo Massimo, prossima fermata Colosseo. Circo Massimo, next stop Colosseo."

Il suonatore passa il suo bicchiere di carta e se ne va. Le mie labbra tornano ad essere frontiere chiuse. "Colosseo, prossima fermata Cavour. Colosseo, next stop Cavour."

Le parole non nascono in fretta come i paesaggi sfrecciati dai finestrini della metro. Per penetrare nel tessuto dell'anima, esigono molto tempo.

"Cavour, prossima fermata Termini. Cavour, next stop Termini."

Sono arrivata. La porta automatica si apre. Esco, senza parole. 